\title{
BMJ Open Surgical care for the aged: a retrospective cross-sectional study of a national surgical mortality audit
}

\author{
Jennifer Allen, ${ }^{1,2}$ John B North, ${ }^{1}$ Arkadiusz Peter Wysocki, ${ }^{3}$ Robert S Ware, ${ }^{2}$ \\ Therese Rey-Conde ${ }^{1}$
}

To cite: Allen J, North JB, Wysocki AP, et al. Surgical care for the aged: a retrospective crosssectional study of a national surgical mortality audit. BMJ Open 2015;5:e006981. doi:10.1136/bmjopen-2014006981

- Prepublication history and additional material is available. To view please visit the journal (http://dx.doi.org/ 10.1136/bmjopen-2014006981).

Received 22 October 2014 Revised 24 February 2015 Accepted 28 February 2015

CrossMark

\footnotetext{
${ }^{1}$ Queensland Audit of Surgical Mortality, Royal Australasian College of Surgeons, Brisbane, Queensland, Australia ${ }^{2}$ School of Population Health, The University of Queensland, Brisbane, Queensland, Australia

${ }^{3}$ Department of Surgery, Logan Hospital, Brisbane, Queensland, Australia
}

Correspondence to Dr Therese Rey-Conde; Therese.Rey-Conde@ surgeons.org

\section{ABSTRACT}

Objectives: It is assumed that increased age signifies increased surgical care. Few surgical studies describe the differences in care provided to older patients compared with younger patients. We aimed to examine the relationships between increasing age, preoperative factors and markers of postoperative care in adults who died in-hospital after surgery in Australia.

Design: This retrospective cross-sectional study extracted data from a national surgical mortality auditan independent, peer-reviewed process.

Setting: From January 2009 to December 2012, 111 public and 61 private Australian hospitals notified the audit of in-hospital deaths after general anaesthetic surgery or if the patient was admitted under a surgeon.

Participants: Notified deaths totalled 19 723. We excluded deaths if patients were brain dead, younger than 17 years or never had an operation $(n=11376)$. From this baseline population, we divided 11201 deaths into three patient age groups: youngest (17-64 years), medium (65-79 years) and oldest ( $\geq 80$ years).

Outcome measures: Univariable and multivariable logistic regression analyses determined the relationships between increasing age and the measured preoperative factors and postoperative variables.

Results: The baseline population's median age was 78 years (IQR 66-85), 43.7\% (4892/11 201) were 80 years or older and $83.4 \%(9319 / 11173)$ had emergency admissions. The oldest group had increased trauma and emergency admissions than the medium and youngest age groups. Seven of the eight measured markers of postoperative care demonstrate strong and significant relationships with increasing age. The oldest group compared with the medium group had decreased rates of: unplanned returns to theatre $(11.2 \%(526 / 4709)$ vs $20.2 \%(726 / 3586))$, unplanned intensive care admissions (16.3\% (545/3350) vs $24.0 \%(601 / 2504)$ ) and treatment in intensive care units (59.7\% (2689/4507) vs $76.7 \%(2754 / 3590))$.

Conclusions: The oldest patients received lower levels of care than the medium and youngest age groups.

\section{INTRODUCTION}

Older patients generally require more surgical services relative to younger patients ${ }^{1}$ as a

\section{Strengths and limitations of this study}

- This study benefited from using data from a large national surgical mortality audit that encompasses all surgical specialties and is multicentred.

- Data were systematically collected using a standard data tool from $90 \%$ of Australian surgeons ensuring a high level of clinical accuracy and very low numbers of loss to follow-up.

- No patient selection bias was present, as the data consisted only of surgical patients that died in-hospital and all surgical deaths are reported to the audit.

- There is a lack of knowledge of the comparator population who had surgery and survived, and therefore the results obtained may not be generalisable to the total surgical population.

- Confounding may be present due to differences in patient characteristics in the three age groups.

consequence of the ageing process and accumulation of chronic disease. ${ }^{2} 3$ As life expectancy and population numbers increase, ${ }^{45}$ so will the requirement for elective and emergency surgical services. ${ }^{1}{ }^{2} \quad{ }^{6-8}$ But surgical care for the older patient is complex. The presence of comorbidities in older patients complicates surgical decision-making, ${ }^{9}$ and is reported to be associated with increases in postoperative complications ${ }^{10} 11$ due to the patients functional, physiological, psychological and social effects. ${ }^{2} 812$

There is a scarcity of data about overall surgical management in older patients. Few surgical studies ${ }^{8} 13$ have described the differences in care provided to older patients compared with that provided to younger patients. Boumendil et $a l^{8}$ found that elderly patients in France had fewer admissions to intensive care than younger patients. Most studies discuss technical surgical procedures on elderly patients, rather than postoperative care for this age group. ${ }^{16}$

Surgical literature is inconsistent in defining old age, with $\geq 65,{ }^{14} \geq 75^{3}$ and $\geq 80$ years $^{8}$ 
all being used. Old age is sometimes used as an independent predictor for surgical morbidity and mortality, but it is reported as having both minimal ${ }^{15}{ }^{16}$ and increased effect ${ }^{2}{ }^{10}$ - possibly because researchers assess the impact of chronological age in a dichotomous fashion using an arbitrary age.

This study aimed to examine the relationships between increasing age, particularly old age, preoperative factors and markers of postoperative care in adult surgical patients who died in-hospital after surgery.

To do this effectively, the audit data were divided into three age groups: youngest (17-64 years), medium (65-79 years) and oldest ( $\geq 80$ years). We describe the differences in preoperative factors and postoperative care provided to these patients who died.

\section{METHODS}

This retrospective cross-sectional study analysed data collected in the Australian and New Zealand Audit of Surgical Mortality (ANZASM), between 1 January 2009 and 31 December 2012. This study covers all of Australia, which has a population of 22.9 million people. ${ }^{17}$ During the study period, there were $\sim 8.4$ million patient separations ${ }^{18}$ from hospital after surgery. No baseline patient population data were available for patients discharged alive from hospital after surgery. The audit data are provided by treating surgeons and 94\% (4268/4540) of Australian surgeons participate in the audit. ${ }^{19}$

Hospitals included in the audit are teaching hospitals that perform surgery that requires an anaesthetist. The full audit process is published ${ }^{20}$ (see online supplementary 1) and briefly described here. Hospitals report in-hospital deaths to the audit, independent of the surgeons, if patients were under the care of a surgeon at the time of death. Reported deaths also include patients palliated postoperatively either at the same hospital or an associated facility. Audit data are systematically collected using a standard data collection form (designed by surgeons) which covers all aspects of surgical care (see online supplementary 2-not for publication). The treating surgeon completes the form, which is de-identified and assessed by external peer surgeons. When required, forms were sent for secondary assessment. Some variables have minor denominator variation as not every question was always answered. Preoperative and postoperative variable answers are based on hospital medical record notes. Surgeon assessors use their professional judgement and clinical evidence when answering subjective questions on the form. Clinical incidents in the audit are defined as areas in healthcare that the surgeon believed could be improved or different; or should have been better; or adverse events.

For the analysis, cases were excluded if forms were pending from the treating surgeon and if the patient: never had surgery, had an American Society of Anaesthetists (ASA) class of VI (organ donor), was admitted for non-operative terminal care and was younger than
17 years of age. We extracted preoperative and postoperative variables from the audit database.

Preoperative variables included: patient age (categorised as youngest, 17-64 years; medium, 65-79 years; and oldest, $\geq 80$ years), gender, type of admission (emergency or elective), the presence of one or more comorbidities, malignancy status, involvement of trauma, ASA class, patient transfer, delay in diagnosis and surgical specialty.

Postoperative markers of care variables included: fluid balance problems, unplanned return to theatre, unplanned intensive care unit (ICU) admission, treated in ICU, clinical incidents, postoperative complication, infection present at death and if retrospectively the surgeon would have done anything differently when managing the patient.

We divided the included audit data into three age groups for several reasons: the discrepant definitions of old age in the literature; our opinion that 65 years is not a true cut-off mark for old age and the biological differences found with increasing age.

Selection bias for reporting of deaths is not present, as deaths are reported independent of the surgeons. Classification bias is not present as death is an end point. Reporting bias may be present for clinical incidents (these are reported retrospectively by the surgeon) and malignancy (a result of missing data, which have been theoretically corrected using multiple imputation).

\section{External validation of the audit data}

The sensitivity of the audit reporting process was externally validated. The deaths reported to the audit were compared with the total number of postoperative mortalities in Australia using Australian Institute of Health and Welfare (AIHW) data. ${ }^{21}$

\section{Ethical approval}

No ethical approval was sought as ANZASM is a protected quality assurance activity under Part VC of the Health Insurance Act 1973 (gazetted August 2011). New Zealand data are excluded in this analysis.

"According to the policy activities that constitute research at the Australian and New Zealand Audit of Surgical Mortality, this work met criteria for operational improvement activities exempt from ethics review."

\section{Statistical analysis}

We calculated ORs for each variable, comparing by age group (ie, youngest vs medium, youngest vs oldest and medium vs oldest). Summary statistics are presented as median (IQR) for continuous variables and frequency (percentage) for categorical variables. The association between continuous variables and age was calculated using the Mann-Whitney $\mathrm{U}$ test. The association between categorical variables and age was calculated with Fisher's exact test.

We calculated the association between age and both preoperative factors and surgical complications using 
both univariable and multivariable logistic regression. In all multivariable models, gender, ASA class, type of admission and presence of malignancy were included as covariables except for the outcomes gender (where gender is excluded as a covariable), admission type (where admission type is excluded as a covariable) and presence of malignancy (where presence of malignancy is excluded as a covariable). Owing to the relatively high amount of missing data for the variable malignancy, multiple imputations using logistic regression was used to impute 20 sets of values. The covariables used in the imputation were gender, type of admission (emergency/ elective), presence of comorbidities (yes/no), whether patient was transferred (yes/no) and whether diagnosis was delayed (yes/no). The univariable association between presence of malignancy and age was assessed using imputed malignancy data. In all multivariable logistic regressions, imputed malignancy data were used. For all other variables, missing items were excluded from analyses. Regression results are presented as OR and $95 \%$ CIs. Significance values were based on two- tailed tests, with $\mathrm{p}<0.05$ considered statistically significant. We performed all analyses using SPSS V.19 (IBM, Armonk, New York, USA) and Stata V.12.1 (StataCorp, College Station, Texas, USA).

\section{RESULTS}

This Australia-wide surgical mortality audit included 61 private and 111 public teaching hospitals that notified the audit of their surgical deaths (this covered $20 \%$ of private hospitals and $99 \%$ of public teaching hospitals). Treating surgeons at the participating hospitals completed forms for their own cases, with a loss to follow-up rate of $2.4 \%$ (482 of 19723 ). Seventy-five per cent $(74.6 \%, 11376 / 15021)$ of the included deaths were considered to be the baseline population of which $98.5 \%$ (11 201 of 15021 ) were categorised by age group. The sensitivity of the reporting process was externally validated by comparison with AIHW data and this showed a $97.6 \%$ correlation-14 659 deaths reported to AIHW compared with 15021 in the audit. To

Figure 1 Flow chart of included mortality data.

†Cases from non-participating surgeons.

$\ddagger$ Cases not returned after 2 years from notification, despite regular reminders, were considered "lots to follow-up".

$\S$ Cases not returned, but $<2$ years, therefore not "lost of follow-up". IIPatients classed as American Society of Anaesthesiologists (ASA) 6 are brain-dead.

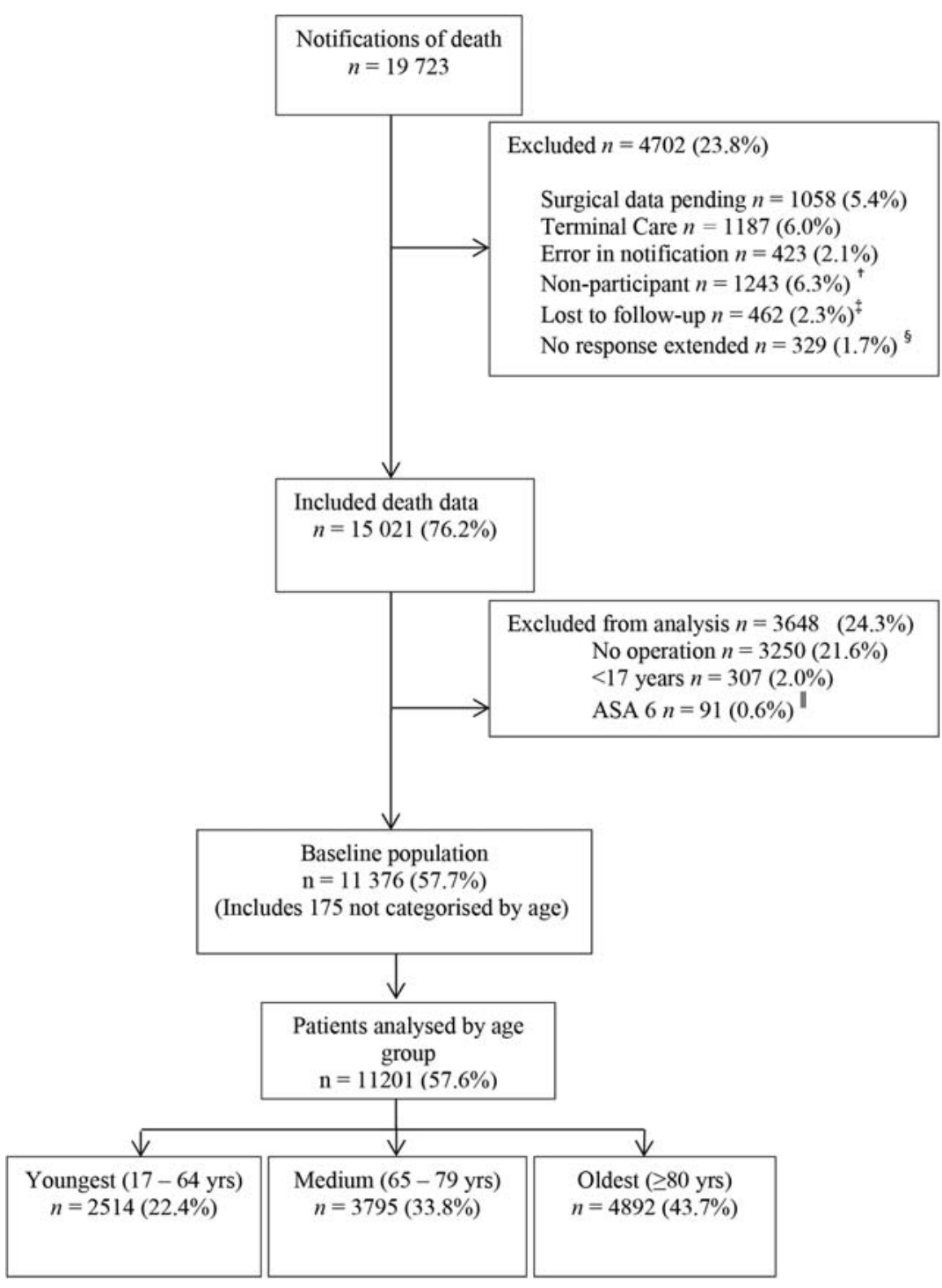




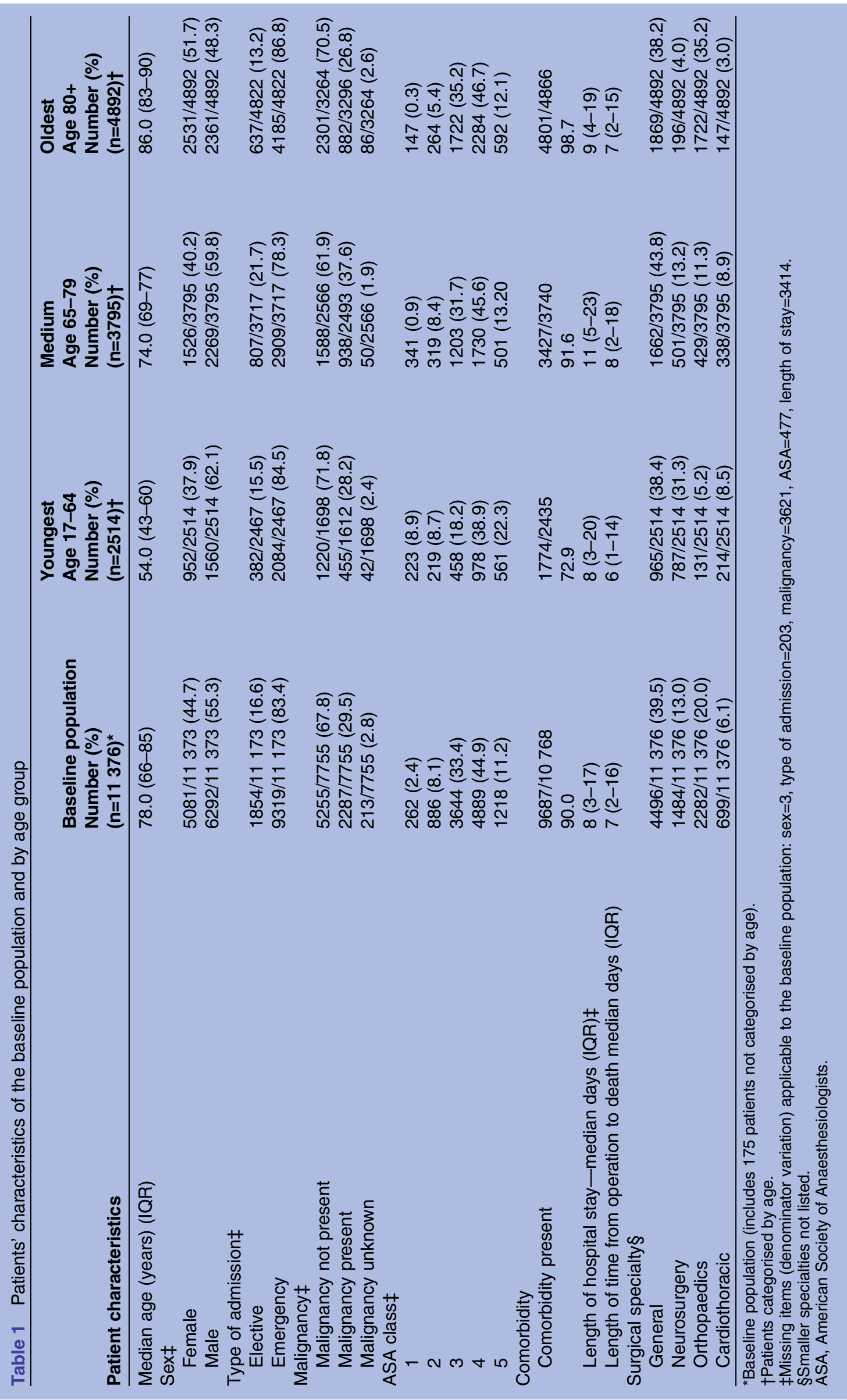


demonstrate the differences in care by age, $22.4 \%$ (2514) were categorised as youngest, 33.9\% (3795) as medium and $43.7 \%$ (4892) as oldest (figure 1).

Patient characteristics for the baseline population and by age group are shown in table 1 . The baseline population's median age was 78 years (IQR 66-85) and less than a quarter of the patients had an elective admission. Nearly half the patients $(44.9 \%, 4889 / 10899)$ had a preoperative incapacitating systemic disease that posed a constant threat to life (ie, ASA4 class). Preoperatively surgeons expected $13 \%$ of the patients would die and $87 \%$ had less than expected risk of death regardless of age.

Patients in the oldest group (table 1) were more likely to be female $(51.7 \%(2531 / 4892))$ compared with the baseline population $(44.7 \%(5081 / 11376))$ as well as the medium (37.9\% (952/2514)) and youngest groups $(40.2 \%(1526 / 3795))$. The prevalence of comorbidities increased with increasing age $(72.9 \%(1774 / 2435)$ vs $91.6 \%(3427 / 3740) \quad$ vs $98.7 \% \quad(4801 / 4866))$. The median length of hospital stay was longest for the medium group ( 11 vs 9 days in the oldest and 8 days in the youngest group).

Analysed preoperative variables (table 2) demonstrated a significant influence of age on gender, emergency admission, presence of malignancy, presence of at least one or more comorbidity and whether the patient was transferred. There was no significant influence of age on evidence of trauma. Adequate discrimination and calibration of all logistic regression models was attained.

Figure 2 illustrates the trends of the analysed markers of postoperative care reported in table 3. More than $90 \%$ of these markers of care showed a statistically significant and strong relationship with increasing age. These findings were evident in both univariable and multivariable analyses. The oldest patients received less care than the medium age group. The oldest patients were less likely to have postoperative complications reported $(33.6 \%, 1621 / 4824)$ than the middle $(40.5 \%$, $1504 / 3711$ ) but more likely than the youngest age group $(30.3 \%, 737 / 2433)$.

\section{DISCUSSION}

We found that decreased patterns of postoperative care occurred in the oldest patients when we divided the patients into three age groups. The differences in care between these groups were statistically significant.

Most of the postoperative markers of care analysed (seven of the eight) demonstrated lower levels of aggressive intervention and resource use. These interventions included admissions to intensive care, when the patients were over 80 years of age. The oldest patients were treated differently from the younger patient groups. Despite being admitted with increased incidence of trauma and more extensive comorbidities, they had fewer postoperative complications diagnosed and shorter length of hospital stay before death than the middle-aged group.

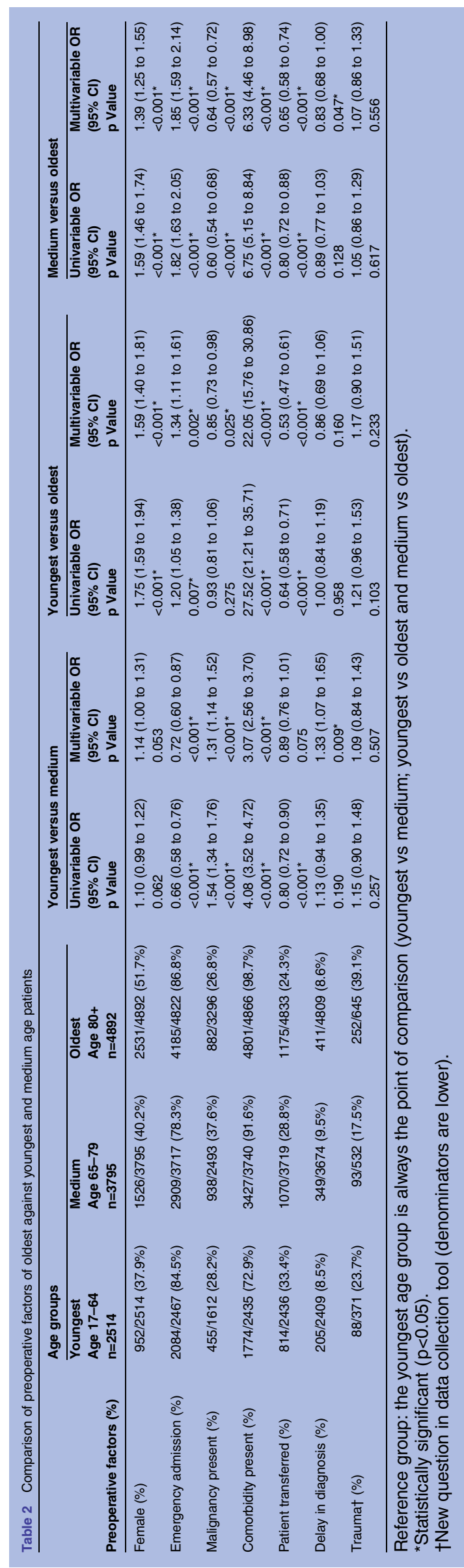


Figure 2 Trends of postoperative factors versus age (2009-2012).
Post-operative factors affected by age

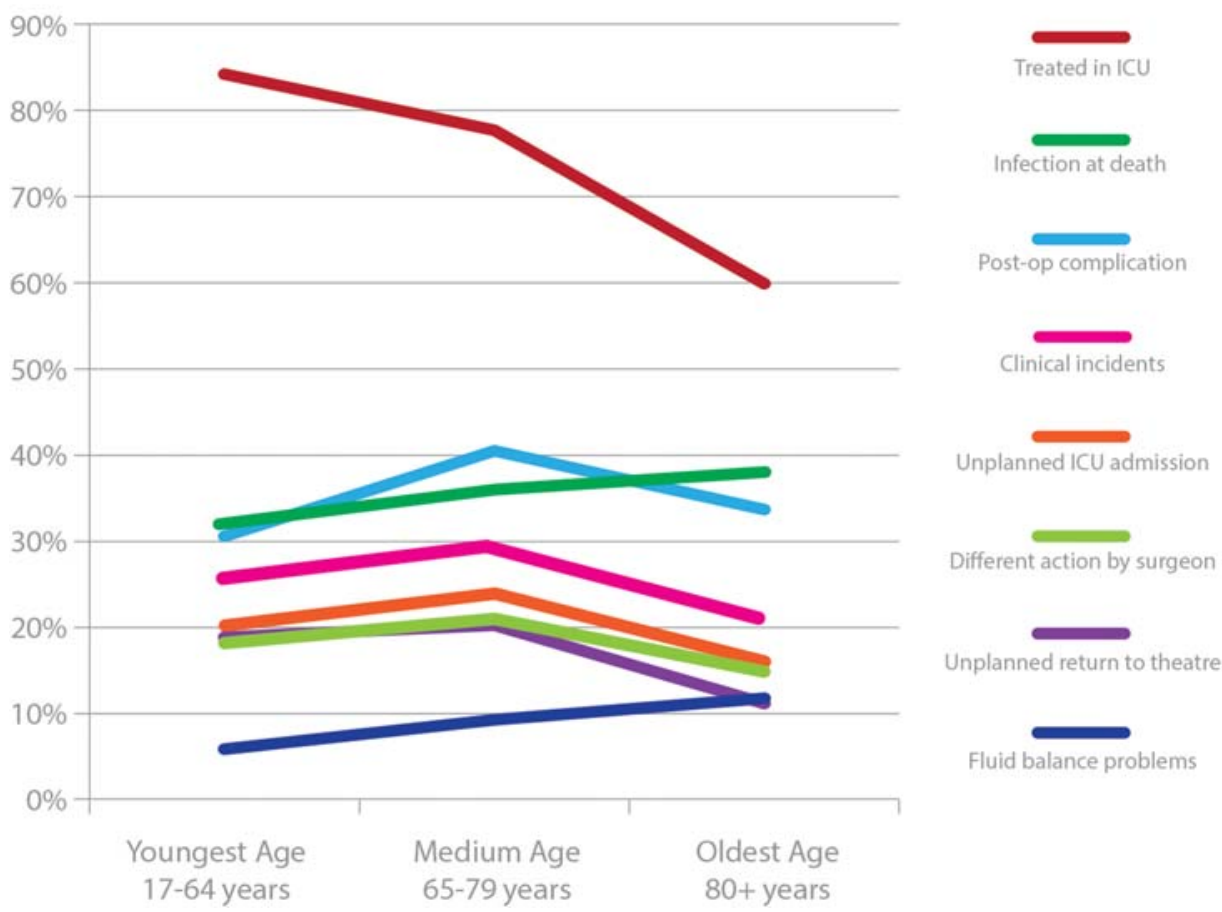

The medium age group (65-79 years) died in a setting of high resource use: highest rates of interhospital transfers, postoperative complications, clinical incidents, unplanned returns to theatre, unplanned intensive care admissions and they had the longest length of hospital stay. Interestingly, this group had the lowest rate of trauma but the highest rate of malignancy, which may have influenced the decisions for ongoing aggressive treatment.

The levels of care in the youngest age group (17-64 years) parallel the levels of care in the oldest age group (80+ years); however, the level of care for both of these age groups was lower than the care given to the medium age group (65-79 years).

Only a few publications have assessed the use of postoperative intensive care for older surgical patients. ${ }^{8} 132223$ Data from the USA, National Surgical Quality Improvement Program, an oft-quoted data base, does not include ICU admission. ${ }^{72}$ Our findings are similar to Boumendil et al who reported decreased admission rates to ICU in patients 80 years and older $(10 \%$ compared with $29 \%$ for patients aged $65-79$ years). Recently, however, Nguyen et $a l^{23}$ reported that the expected benefits of medical or unplanned surgical ICU admissions of patients aged 80 years and older are particularly weak and that the admission of these categories of patients to ICU is questionable. Admission to an ICU is often rationalised due to high operational costs whatever the age group, ${ }^{25}$ and may be influenced by the presence of malignancy and its staging. ${ }^{26}$ Because our data showed that the oldest group had the lowest prevalence of malignancy and the lowest admission to ICU, it is clear that factors other than the diagnosis influenced the decision for ICU admission.

Comorbidities in older patients are reported as being associated with increases in postoperative complications. ${ }^{10}{ }^{11}$ The management decisions with these patients may be influenced by the patients' functional, physiological, psychological and social conditions. ${ }^{2} 812$ But our data showed that detection of postoperative complications was lowest in the oldest age group, despite virtually all having multiple comorbidities. Our data suggest that there may be a culture of less intensive investigation, monitoring and possible failure to intervene in the elderly group. From our data, it is difficult to determine the appropriateness of these levels of care because the study is based on after death self-reporting. Guidelines and scoring systems have been developed to ascertain the point at which 'intervention' is appropriate, that is, when surgery or aggressive intervention is futile. The use of scoring systems is rarely quoted by selfreporting surgeons in the data of this mortality audit. This suggests that such systems should be used in the mostly elderly surgical population on an everyday basis.

The greatest strength of this study is its large sample size. This ensured the robustness of the data and the ability to make broad statements concerning the findings. It encompassed all surgical specialties, is multicentred covering approximately $70 \%$ of Australian surgical hospitals-both private and public. ${ }^{27}$ Over $90 \%$ of Australian surgeons participated ${ }^{19}$ as part of the Continuing Professional Development Program of the Royal Australasian College of Surgeons. Data were systematically collected using a standard data tool. 


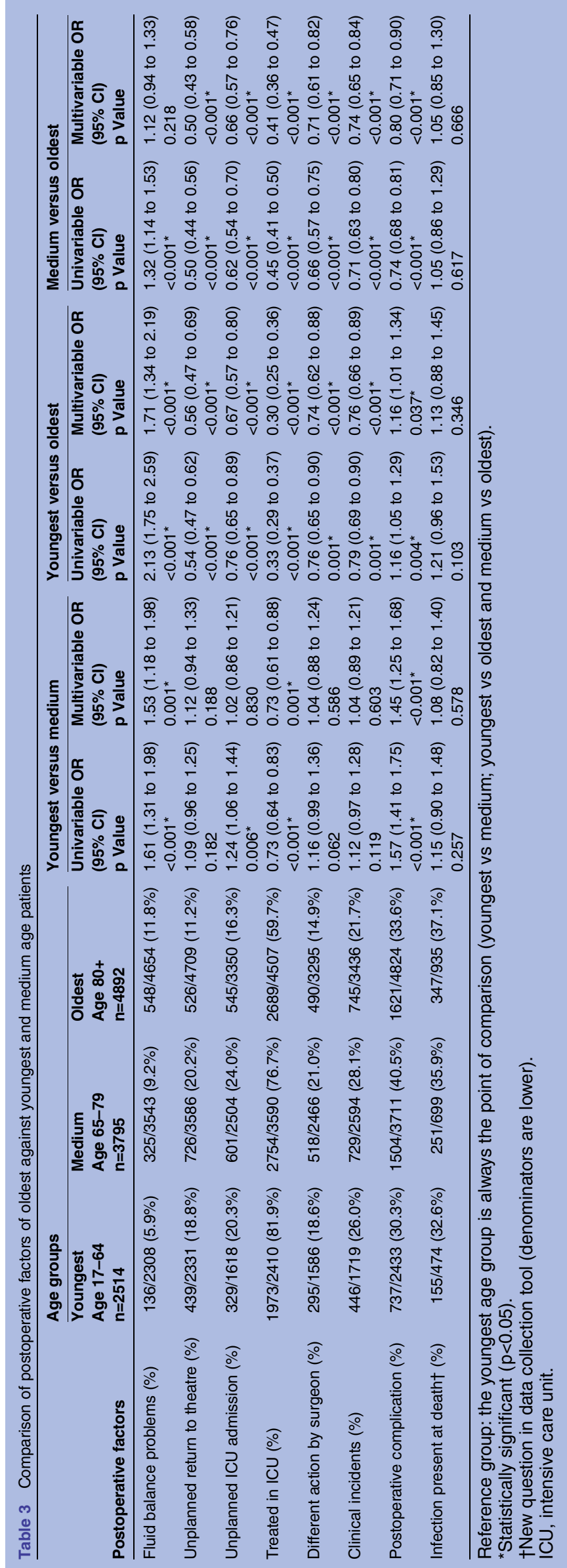

No patient selection bias was present, as the cohort consisted only of surgical patients who died in-hospital, and all in-hospital surgical deaths are reported to the audit. The sensitivity of deaths reported to the audit is high and was externally validated against those reported to AIHW over the same period. Reported audit deaths were higher than the AIHW which included perioperative deaths, while the audit included all patients who died while admitted under a surgeon's care even if no operations were performed. Surgeons self-reported the dataensuring a high level of clinical accuracy. The numbers of forms that were 'lost to follow-up' were very low. Data entry accuracy was checked and conformed to recognised criteria. ${ }^{28}$ We addressed confounding by using multivariable analysis.

Notwithstanding the above, this study has several limitations. These include a database designed to audit inhospital surgical deaths that was not designed for clinical research. The database allows minor denominator variation but includes in-hospital surgically related deaths only and no morbidity data. The design excludes patients who had local anaesthesia, patients who had surgery and survived, or who were subsequently transferred to an external palliation facility. Therefore, there is a lack of knowledge of the comparator population who had surgery and survived. Moreover, there were no frailty assessment scores or other mental or functional assessment modalities. Confounding may be present due to differences of patients within the surgical subspecialties. The collection of comorbidities prevalent in each surgical subspecialty was not included in the analysis. The reasons why care was given or withheld were not assessed. The variable 'detection of postoperative complications' may be subject to bias and may well be under-reported in hospitals. Reporting bias could be present due to surgeons' self-reporting, but in effect, the ANZASM process limits this, because of the random review of $14 \%$ of cases. These reviews compare the surgeons' self-reporting with hospital medical records, and discrepancies are very rare.

We should look for the explanations for our findings outside of the surgical mortality database. We postulate that in the oldest age group, care may be less aggressive, or appropriately scaled down. This may be due to expectations of poor outcome, concerns of futility of care or settings of competing comorbidities. Also perceived future quality of life issues may influence decisions by care givers, especially in the presence of malignancy, which in this data set at least $30 \%$ were missing. The surgical audit does not collect variables to test the validity of these observations.

These results need to be confirmed with future studies to determine: the treatment and admission of older surgical patients in ICUs, the prompt detection of postoperative complications in the oldest and whether the levels of intervention in the oldest are clinically appropriate. Our unexpected findings showing that the medium age group died with the highest level of care also require further study. 


\section{CONCLUSION}

This national mortality audit data uniquely identified complex relationships between advancing age and postoperative care in adults who died following surgery. We showed strong decreases in resource use in the oldest group. We demonstrated a decrease in levels of postoperative care in patients 80 years or older. These findings may indicate a willingness to offer an operation on presentation, but early withdrawal of treatment if complications occur-rather than initial instigation of palliative care. As a result, surgical costs may increase at a lower rate than expected if older people continue to have fewer complex postoperative interventions.

Acknowledgements The authors acknowledge: the Queensland Department of Health's ongoing support of the audit, the Patient Safety Units' Senior Director, Kirstine Sketcher-Baker and Assistant Director, Erin Finn and the surgeons of Queensland. The authors appreciate Dr John Quinn, the critique of the manuscript. They appreciate the dedication to the audit by: Professor Guy Maddern, Associate/Professor Wendy Babidge, Mr Gordon Guy, Ms Sonya Faint and Ms Kyrsty Webb, and all participating hospitals and their staff.

Contributors All authors have contributed towards the publishing of this manuscript. JA (25\%) and JBN (20\%) contributed to the concept of the hypothesis, interpretation of the data, drafting and revising the article including intellectual content as well as final approval of the version for publication. APW (15\%) contributed to the interpretation of the data, critically revising the included intellectual content and final approval of the version for publication. RSW (15\%) contributed to the concept of the hypothesis, statistical data analysis and writing of the content. TR-C (25\%) contributed to the concept of the hypothesis, analysis and interpretation of the data, drafting and revising the article including intellectual content as well as final approval of the version for publication.

Funding This audit is supported by the Queensland Department of Health, Brisbane, Australia; and is managed by the Royal Australasian College of Surgeons.

\section{Competing interests None declared.}

Provenance and peer review Not commissioned; externally peer reviewed.

Data sharing statement The corresponding author is able to provide de-identified data for this study if requested.

Open Access This is an Open Access article distributed in accordance with the Creative Commons Attribution Non Commercial (CC BY-NC 4.0) license, which permits others to distribute, remix, adapt, build upon this work noncommercially, and license their derivative works on different terms, provided the original work is properly cited and the use is non-commercial. See: http:// creativecommons.org/licenses/by-nc/4.0/

\section{REFERENCES}

1. Etzioni DA, Liu JH, Maggard MA, et al. The aging population and its impact on the surgery workforce. Ann Surg 2003;238:170-7.

2. Turrentine FE, Wang $\mathrm{H}$, Simpson VB, et al. Surgical risk factors, morbidity, and mortality in elderly patients. J Am Coll Surg 2006;203:865-77.

3. Bentrem DJ, Cohen ME, Hynes DM, et al. Identification of specific quality improvement opportunities for the elderly undergoing gastrointestinal surgery. Arch Surg 2009;144:1013-20.

4. Wang $\mathrm{H}$, Dwyer-Lindgren L, Lofgren $\mathrm{KT}$, et al. Age-specific and sex-specific mortality in 187 countries, 1970-2010: a systematic analysis for the Global Burden of Disease Study 2010. Lancet 2012;380:2071-94.

5. Australian Bureau of Statistics. 3222.0-Population Projections, Australia, 2012 (base) to 2101 Canberra, Australia 2013. http://www.
abs.gov.au/ausstats/abs@.nsf/Lookup/3222.0main+features52012\% 20(base)\%20to\%202101 (accessed 12 Jun 2014).

6. Liu JH, Etzioni DA, O'Connell JB, et al. The increasing workload of general surgery. Arch Surg 2004;139:423-8.

7. Hamel MB, Henderson WG, Khuri SF, et al. Surgical outcomes for patients aged 80 and older: morbidity and mortality from major noncardiac surgery. J Am Geriatr Soc 2005;53:424-9.

8. Boumendil A, Aegerter P, Guidet B. Treatment intensity and outcome of patients aged 80 and older in intensive care units: a multicenter matched-cohort study. J Am Geriatr Soc 2005;53:88-93.

9. Cuvillon P, Nouvellon E, Marret E, et al. American Society of Anesthesiologists' physical status system: a multicentre francophone study to analyse reasons for classification disagreement. Eur $J$ Anaesthesiol 2011;28:742-7.

10. Massarweh NN, Legner VJ, Symons RG, et al. Impact of advancing age on abdominal surgical outcomes. Arch Surg 2009;144:1108-14.

11. Wu CH, Cheng RM, Tsai HC, et al. How comorbidities and preoperative expenditures correlate with postoperative adverse outcomes. Am J Manag Care 2012;18:e405-415.

12. Lyon C, Clark DC. Diagnosis of acute abdominal pain in older patients. Am Fam Physician 2006;74:1537-44.

13. Story DA, Leslie K, Myles PS, et al. Complications and mortality in older surgical patients in Australia and New Zealand (the REASON study): a multicentre, prospective, observational study. Anaesthesia 2010;65:1022-30.

14. Duron JJ, Duron E, Dugue T, et al. Risk factors for mortality in major digestive surgery in the elderly: a multicenter prospective study. Ann Surg 2011;254:375-82.

15. Lau D, Granke K, Olabisi R, et al. Carotid endarterectomy in octogenarian veterans: does age affect outcome? A single-center experience. Am J Surg 2005;190:795-9.

16. Lidsky ME, Thacker JKM, Lagoo-Deenadayalan SA, et al. Advanced age is an independent predictor for increased morbidity and mortality after emergent surgery for diverticulitis. Surgery 2012;152:465-72.

17. Australian Bureau of Statistics. 3101.0-Australian Demographics: Australian Government; Dec 2012. [updated 20 Jun 2013]. http:// www.abs.gov.au/ausstats/abs@.nsf/mf/3101.0 (accessed 29 Jul 2013).

18. Australian Institute of Health and Welfare 2013. Australia's hospitals at a glance 2010-11: Australian Institute of Health and Welfare. http://www.aihw.gov.au/haag10-11/admitted-patient-care-surgery/ (accessed 2 Sep 2013)

19. Royal Australasian College of Surgeons. Australian and New Zealand Audit of Surgical Mortality National Report 2011. http:// www.surgeons.org/media/18835539/rpt_2013-02-15_anzasm annual_report_2011_updated.pdf (accessed 30 Jul 2013).

20. Raju RS, Guy GS, Majid AJ, et al. The Australian and New Zealand audit of surgical mortality-birth, deaths, and carriage. Ann Surg 2015;261:304-8.

21. Australian Institute of Health and Welfare. Australian hospital statistics 2012-13. Health services series no. 54. Cat. no. HSE 145. Secondary Australian hospital statistics 2012-13. 2014. http://www. aihw.gov.au/publication-detail/?id=60129546922

22. McNicol L, Story DA, Leslie K, et al. Postoperative complications and mortality in older patients having non-cardiac surgery at three Melbourne teaching hospitals. Med J Aust 2007;186:447-52.

23. Nguyen YL, Angus DC, Boumendil A, et al. The challenge of admitting the very elderly to intensive care. Ann Intensive Care 2011;1:29.

24. American College of Surgeons National Surgical Quality Improvement Program. User guide for the 2011 Participant Use Data File. Chicago, IL: American College of Surgeons: American College of Surgeons. http://site.acsnsqip.org/wp-content/uploads/2012/03/ 2011-User-Guide_Final.pdf (accessed 9 May 2013).

25. Pintado M-C, Villa P, Gonzalez-Garcia N, et al. Characteristics and outcomes of elderly patients refused to ICU. Sci World J 2013;2013:7.

26. Soares M, Salluh Jl. Prognostic factors in cancer patients in the intensive care unit. Indian J Crit Care Med 2007;11:19-24.

27. Australian Institute of Health and Welfare. Rise in hospital admissions for older Australians: Australian Government. http://www.aihw.gov.au/ media-release-detail/?id=60129543135 (accessed 9 May 2013).

28. Russell EM, Bruce J, Krukowski ZH. Systematic review of the quality of surgical mortality monitoring. Br J Surg 2003:90:527-32. 